\title{
The role of string-like, supramolecular assemblies in reentrant supernematic liquid crystals
}

\author{
Marco G. Mazza ${ }^{\dagger}$, Manuel Greschek ${ }^{\dagger}$, Rustem Valiullin*, and Martin Schoen ${ }^{\dagger, \ddagger}$ \\ ${ }^{\dagger}$ Stranski-Laboratorium für Physikalische und Theoretische Chemie, \\ Technische Universität Berlin, Straße des 17. Juni 135, 10623 Berlin, Germany, \\ * Institut für Experimentelle Physik I, Universität Leipzig, Linnéstraße 5, 04103 Leipzig, Germany, \\ ${ }^{\ddagger}$ Department of Chemical and Biomolecular Engineering, \\ North Carolina State University, 911 Partners Way, Raleigh, NC 27695, U.S.A.
}

(Dated: August 6, 2018)

\begin{abstract}
Using a combination of isothermal-isobaric Monte Carlo and microcanonical molecular dynamics we investigate the relation between structure and self-diffusion in various phases of a model liquid crystal using the Gay-Berne-Kihara potential. These molecules are confined to a mesoscopic slitpore with atomically smooth substrate surfaces. As reported recently [see M. G. Mazza et al., Phys. Rev. Lett. 105, 227802 (2010)], a reentrant nematic (RN) phase may form at sufficiently high pressures/densities. This phase is characterized by a high degree of nematic order and a substantially enhanced self-diffusivity in the direction of the director $\widehat{\boldsymbol{n}}$ which exceeds that of the lower-density nematic and an intermittent smectic A phase by about an order of magnitude. Here we demonstrate that the unique transport behavior in the RN phase may be linked to a confinementinduced packing effect which causes the formation of supramolecular, string-like conformations. The strings consist of several individual molecules that are capable of travelling in the direction of $\widehat{\boldsymbol{n}}$ as individual "trains" consisting of chains of molecular "cars". Individual trains run in parallel and may pass each other at sufficiently high pressures.
\end{abstract}

PACS numbers: 61.30.Hn,66.10.C-,64.70.M-,61.30.Gd

\section{INTRODUCTION}

A thermodynamic phase is called "reentrant" when an order parameter varies nonmonotonicaly with the thermodynamic field driving the transition. Reentrancy is ubiquitous in the physics of thermal many-particle systems. It arises under quite disparate physical conditions encountered, for example, in quantum gases [1], two-dimensional charged colloids [2], or relativistic scalar field models 3]. As far as soft matter is concerned reentrancy has been reported for self-assembled supramolecular structures [4, 5], wetting phenomena at oleophilic surfaces [6] , and novel discotic and calamitic liquid crystals [7]. In fact, since the first observation of reentrant nematic (RN) phases in a seminal paper by Cladis [8] reentrancy in liquid crystals seems to have received most of the attention. Liquid crystals offer rich and complex phase diagrams due to the characteristic interplay of translational and rotational degrees of freedom. For example, reentrant phase transitions have been reported for the isotropic phase of mixtures of discotic liquid crystals [9], the ferroelectric transition in smectic $\mathrm{C}$ phases [10], the cholesteric-to-blue phase transition in chiral liquid crystals [1], and for nematic (N) phases [12].

After the work of Cladis [8] on binary mixtures, and later in pure compounds at high pressure and in the supercooled state [13], Hardouin et al. 14] showed the occurrence of RN phases also for pure compounds at atmospheric pressure. For comprehensive reviews the reader should consult Refs. 12 and 15. Whereas most earlier work on reentrancy of phase transitions in liquid crystals is experimental in nature comparatively little atten- tion has been paid to this fascinating phenomenon from a theorist's point of view. Early theoretical approaches have mainly considered dimer models and frustration effects 15 18]. Ferrarini et al. consider the role of association and isomerization equilibria for the formation of RN phases within a mean-field-type of theory [19]. The most recent theoretical study employs isothermalisobaric and canonical ensemble Monte Carlo (MC) simulations to investigate the nature of the smectic $\mathrm{A}(\mathrm{smA})$ $\mathrm{RN}$ phase transition for a bulk system of hard ellipsoids with square-well attraction 20] where earlier theoretical studies are briefly reviewed, too. Unfortunately, the model employed in Ref. 20 is somewhat artificial in assuming that the ellipsodal molecules are always oriented in a perfectly parallel fashion such that all rotational degrees of freedom are "frozen" irrespective of the thermodynamic conditions. Therefore, this study seems only of limited use to elucidate properties of RN phases at a molecular level. Moreover, the authors do not consider dynamic features of $\mathrm{RN}$ phases.

Despite the variety of systems and thermodynamic conditions under which reentrancy in liquid-crystalline materials arises comparatively little attention has been paid to the dynamics of reentrant phases. To the best of our knowledge the only study of the dynamics of reentrant liquid-crystalline phases has recently been conducted by ourselves and focuses on self-diffusion in the $\mathrm{RN}$ phase as a specific case 21]. In that work we considered rod-like mesogens where the interaction between a pair of rods takes into account both a realistic shape of the molecules and the orientation dependence of their interaction. Unlike de Miguel and Martín del Río [20] we allowed our molecules to rotate as freely as the spe- 
cific thermodynamic conditions permit. We could then demonstrate 21 that on account of the high degree of nematic order diffusion of molecules in the direction of the nematic director may be enhanced by about an order of magnitude over that characteristic of lower-density $\mathrm{N}$ or smA phases. The combination of a high degree of nematic order with a substantially enhanced self-diffusivity in the direction of the nematic director prompted us to refer to liquid crystals in the RN phase as "supernematics" 21]. Enhanced self-diffusivity in the RN phase bears a striking similarity to "levitation" in zeolites. Experiments have demonstrated the self-diffusion constant to pass through a maximum when the size of a diffusant molecule is comparable to the pore size, that is enhanced diffusivity is observed as a result of severe confinement to tiny voids in the host material (see, for example, Ref. 22).

For liquid crystalline materials we could explain this unusually enhanced self-diffusion quantitatively in terms of a severely reduced rotational configurational entropy 21]. The relation between self-diffusion and structural features of the RN phase has not been explored. In fact, as we shall show in this work the $\mathrm{RN}$ phase is characterized by unique conformations of entire groups of its molecules to which we refer as "strings". Strings may be thought of as train-like arrangements of several molecules that stay together as molecular "cars" and diffuse as a supramolecular entity. As we argue below it is the fairly high pressure that keeps the trains together and which allows them to overcome the attractive interactions between molecules pertaining to neighboring trains. From the experimental side there also exists some evidence suggesting unique dynamic features of the RN phase. For example, distinct differences in the molecular dynamics in the $\mathrm{N}$ and $\mathrm{RN}$ phases can be concluded from corresponding changes in the nuclear magnetic resonance (NMR) relaxation times reported in Refs. 23 26. However, a more detailed account of the present experimental state of the art is postponed until Sec. IV]

Last but not least we note that an improved understanding of the dynamics of liquid crystals is not only of academic interest but may also be important from a purely practical point of view. For example, by pumping liquid crystalline material through vesiscles, that maybe perceived as confined geometries, a spider is capable to spin silk with unsual materials properties that result essentially from the transport of the liquid crystal. Materials with properties comparable to spider silk cannot be produced in the laboratory to date (see Fig. 2 in Ref. 27.

The remainder of our manuscript is organized as follows. Our model liquid crystal is introduced in Sec. II whereas Sec. III is given to a presentation of our findings. In Sec. IV we summarize our results, discuss their physical origin, and put them in a broader experimentaltheoretical context. Specifically, we rationalize the relation between the formation of supramolecular strings and enhanced self-diffusion in the RN phase. Details about the theoretical background of our simulations can be found in Appendix $\mathrm{A}$

\section{SIMULATIONAL DETAILS}

\section{A. Model potentials}

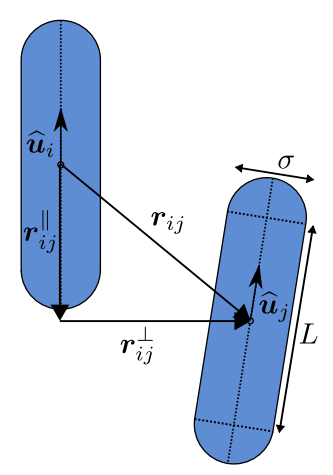

FIG. 1: Cartoon of a pair of spherocylinders of length $L+\sigma$ where $\widehat{\boldsymbol{u}}_{i}$ and $\widehat{\boldsymbol{u}}_{j}$ describe the orientation of molecules $i$ and $j$ of that pair in a space-fixed Cartesian coordinate system; $\boldsymbol{r}_{i j}$ is their center-of-mass distance vector which can be decomposed into components (anti-)parallel $\left(\boldsymbol{r}_{i j}^{\|}\right)$and perpendicular $\left(\boldsymbol{r}_{i j}^{\perp}\right)$ with $\widehat{\boldsymbol{u}}_{i}$, respectively, as indicated.

As in 21] we employ a fluid composed of $N$ spherocylinders of length $L$ and diameter $\sigma$ capped at both ends by a hemisphere of the same diameter $2 R=\sigma$ (see Fig. (1). For this system the Hamiltonian may be cast as

$$
H=\sum_{i=1}^{N} \frac{p_{i}^{2}}{2 m}+\sum_{i=1}^{N} \sum_{\alpha=x, y} \frac{\mathcal{L}_{i \alpha}^{2}}{\mathcal{I}}+U(\boldsymbol{R}, \boldsymbol{\Gamma})
$$

where $\boldsymbol{P} \equiv\left(\boldsymbol{p}_{1}, \boldsymbol{p}_{2}, \ldots, \boldsymbol{p}_{N}\right)$ are the linear momenta conjugate to the set of center-of-mass positions $\boldsymbol{R} \equiv$ $\left(\boldsymbol{r}_{1}, \boldsymbol{r}_{2}, \ldots, \boldsymbol{r}_{N}\right)$ and $\boldsymbol{\Gamma} \equiv\left(\boldsymbol{\gamma}_{1}, \boldsymbol{\gamma}_{2}, \ldots, \boldsymbol{\gamma}_{N}\right)$ are the Euler angles specifying the orientation of each molecule. For the special case of spherocylinders with two rotational degrees of freedom, $\gamma_{i}=\left(\theta_{i}, \phi_{i}\right)$ is a two-dimensional vector composed of two of the three Euler angles. In Eq. (1), $\mathcal{L}_{i \alpha}$ is the component of the angular momentum referred to the body-fixed principal axis $\alpha$ of molecule $i$, $m$ is the molecular mass, and

$$
\mathcal{I}=\frac{3 m}{3 L+R}\left(\frac{8 R^{3}}{15}+\frac{3 R^{2} L}{4}+\frac{R L^{2}}{3}+\frac{L^{3}}{12}\right)
$$

is the moment of inertia of a spherocylinder.

In a computer simulation [regardless of whether Monte Carlo (MC) or molecular dynamics (MD) is employed] the key quantity is the configurational potential energy $U(\boldsymbol{R}, \widehat{\boldsymbol{U}})$ of the system [see, for example, Eqs. (A2), (A5b), or [A7] ] where $\widehat{\boldsymbol{U}} \equiv\left(\widehat{\boldsymbol{u}}_{1}, \widehat{\boldsymbol{u}}_{2}, \ldots, \widehat{\boldsymbol{u}}_{N}\right)$ is a set of unit vectors specifying the orientation of the molecules in a three-dimensional space-fixed Cartesian coordinate system (see Fig. 1). Elements of the set $\widehat{\boldsymbol{U}}$ are related to corresponding elements of $\boldsymbol{\Gamma}$ via

$$
\mathrm{d} \widehat{\boldsymbol{u}}_{i}=\sin \theta_{i} \mathrm{~d} \theta_{i} \mathrm{~d} \phi_{i}, \quad i=1, \ldots, N
$$


In the general case of a confined fluid $U(\boldsymbol{R}, \widehat{\boldsymbol{U}})$ can be decomposed into a fluid-fluid (ff) and a fluid-substrate (fs) contribution. Assuming pairwise additive interactions the former can be cast as

$$
U_{\mathrm{ff}}(\boldsymbol{R}, \widehat{\boldsymbol{U}})=\frac{1}{2} \sum_{i=1}^{N} \sum_{j=1 \neq i}^{N} u_{\mathrm{ff}}\left(d_{i j}^{\mathrm{m}}\right)
$$

whereas the latter may be expressed as

$$
U_{\mathrm{fs}}(\boldsymbol{R}, \widehat{\boldsymbol{U}})=\sum_{k=1}^{2} \sum_{i=1}^{N} u_{\mathrm{fs}}\left(d_{i k}^{\mathrm{m}}\right)
$$

In Eqs. (4) and (5), $u_{\mathrm{ff}}$ and $u_{\mathrm{fs}}$ represent the specific model potential adopted to describe the intermolecular interaction between two rodlike molecules and between a rodlike molecule and a planar, structureless solid substrate, respectively. In this work we adopt the so-called Gay-Berne-Kihara (GBK) model [28]. In the GBK model the interaction between a pair of spherocylinders depends on the relative molecular orientation through the function $d_{i j}^{\mathrm{m}}\left(\boldsymbol{r}_{i j}, \widehat{\boldsymbol{u}}_{i}, \widehat{\boldsymbol{u}}_{j}\right)$ which depends on the center-of-mass distance vector $\boldsymbol{r}_{i j} \equiv \boldsymbol{r}_{i}-\boldsymbol{r}_{j}$ and the orientations of molecules $i$ and $j$ such that $d_{i j}^{\mathrm{m}}$ is actually the minimum distance between that pair of molecules. More specifically,

$$
u_{\mathrm{ff}}=4 \varepsilon_{\mathrm{ff}}\left(\widehat{\boldsymbol{r}}_{i j}, \widehat{\boldsymbol{u}}_{i}, \widehat{\boldsymbol{u}}_{j}\right)\left[\left(\frac{\sigma}{d_{i j}^{\mathrm{m}}}\right)^{12}-\left(\frac{\sigma}{d_{i j}^{\mathrm{m}}}\right)^{6}\right]
$$

where $\widehat{\boldsymbol{r}} \equiv \boldsymbol{r} / r$ and $r \equiv|\boldsymbol{r}|$. In Eq. (6) the function

$$
\begin{aligned}
\varepsilon_{\mathrm{ff}}\left(\widehat{\boldsymbol{r}}_{i j}, \widehat{\boldsymbol{u}}_{i}, \widehat{\boldsymbol{u}}_{j}\right)= & \epsilon_{\mathrm{ff}}\left\{1-\frac{\chi^{\prime}}{2}\left[\frac{\left(\widehat{\boldsymbol{r}}_{i j} \cdot \widehat{\boldsymbol{u}}_{i}+\widehat{\boldsymbol{r}}_{i j} \cdot \widehat{\boldsymbol{u}}_{j}\right)^{2}}{1+\chi^{\prime} \widehat{\boldsymbol{u}}_{i} \cdot \widehat{\boldsymbol{u}}_{j}}+\right.\right. \\
& \left.\left.\frac{\left(\widehat{\boldsymbol{r}}_{i j} \cdot \widehat{\boldsymbol{u}}_{i}-\widehat{\boldsymbol{r}}_{i j} \cdot \widehat{\boldsymbol{u}}_{j}\right)^{2}}{1-\chi^{\prime} \widehat{\boldsymbol{u}}_{i} \cdot \widehat{\boldsymbol{u}}_{j}}\right]\right\}^{2} \\
& \times \frac{1}{\sqrt{1-\chi^{2}\left(\widehat{\boldsymbol{u}}_{i} \cdot \widehat{\boldsymbol{u}}_{j}\right)^{2}}}
\end{aligned}
$$

describes the orientation dependence of the anisotropy strength where the parameters $\chi$ and $\chi^{\prime}$ are given by

$$
\begin{aligned}
\chi & \equiv \frac{\kappa^{2}-1}{\kappa^{2}+1} \\
\chi^{\prime} & \equiv \frac{\sqrt{\kappa^{\prime}}-1}{\sqrt{\kappa^{\prime}}+1}
\end{aligned}
$$

In these latter two expressions parameters $\kappa=L+1(L$ in units of $\sigma$ ) and $\kappa^{\prime}$ may be thought of as the aspect ratio of a spherocylinder and the interaction strength for a side-side relative to an end-end configuration of a pair of spherocylinders, respectively. This can be seen from various plots in Fig. 22 indicating that the potential minimum depends strongly on the molecular orientation such that a side-side configuration of a molecular pair is the energetically most favored one. One also notices from Fig. 2

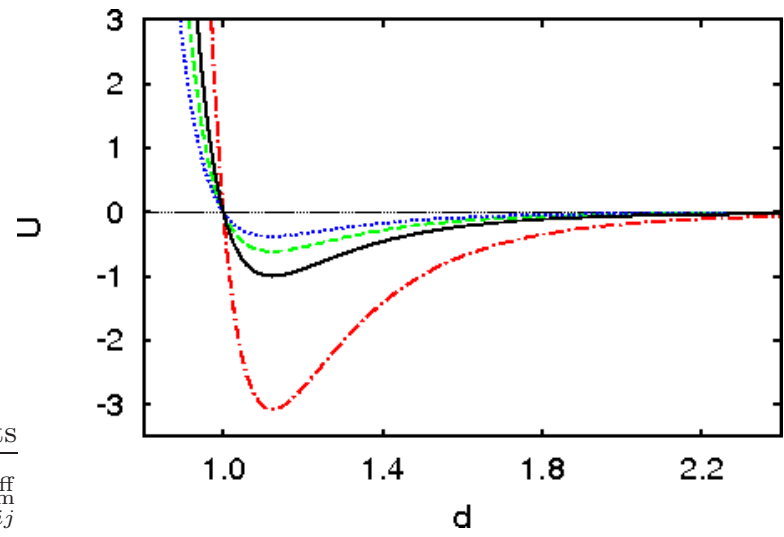

FIG. 2: (Color online) Plots of $u_{\mathrm{ff}}$ as functions of minimum distance $d_{i j}^{\mathrm{m}}\left(\boldsymbol{r}_{i j}, \widehat{\boldsymbol{u}}_{i}, \widehat{\boldsymbol{u}}_{j}\right)$ between a pair of spherocylinders in units of $\epsilon_{\mathrm{ff}}$ and $\sigma$, respectively, and for various relative orientations such as side-side $\left(\widehat{\boldsymbol{r}}_{i j} \cdot \widehat{\boldsymbol{u}}_{i}=\widehat{\boldsymbol{r}}_{i j} \cdot \widehat{\boldsymbol{u}}_{j}=0, \widehat{\boldsymbol{u}}_{i} \cdot \widehat{\boldsymbol{u}}_{j}=1\right)$ $(-\cdot-)$, end-end $\left(\widehat{\boldsymbol{r}}_{i j} \cdot \widehat{\boldsymbol{u}}_{i}=\widehat{\boldsymbol{r}}_{i j} \cdot \widehat{\boldsymbol{u}}_{j}=1, \widehat{\boldsymbol{u}}_{i} \cdot \widehat{\boldsymbol{u}}_{j}=1\right)(\mathbf{- - -})$, T-shaped $\left(\widehat{\boldsymbol{r}}_{i j} \cdot \widehat{\boldsymbol{u}}_{i}=0, \widehat{\boldsymbol{r}}_{i j} \cdot \widehat{\boldsymbol{u}}_{j}=1, \widehat{\boldsymbol{u}}_{i} \cdot \widehat{\boldsymbol{u}}_{j}=0\right)(\cdots)$, and $\operatorname{crossed}\left(\widehat{\boldsymbol{r}}_{i j} \cdot \widehat{\boldsymbol{u}}_{i}=0, \widehat{\boldsymbol{r}}_{i j} \cdot \widehat{\boldsymbol{u}}_{j}=0, \widehat{\boldsymbol{u}}_{i} \cdot \widehat{\boldsymbol{u}}_{j}=0\right)$ (—) (see Fig. (1).

that both the location of the potential minimum as well as the range of distances over which $u_{\mathrm{ff}}$ becomes repulsive depend very little on the intermolecular orientation as one would expect on account of the spherocylindrical shape of the molecules.

In a similar spirit, $d_{i k}^{\mathrm{m}}\left(z_{i k}, \widehat{\boldsymbol{u}}_{i}\right)$ introduced in Eq. (5) is the minimum distance between a spherocylinder with orientation $\widehat{\boldsymbol{u}}_{i}$ whose center of mass is at a vertical distance $z_{i k}=z_{i} \pm s_{z} / 2$ from the lower $(k=1)$ and upper $(k=2)$ substrate located at $-s_{\mathrm{z}} / 2$ and $+s_{\mathrm{z}} / 2$ along the $z$-axis of a space-fixed Cartesian coordinate system, respectively. Assuming the substrates to be planar, $d_{i k}^{\mathrm{m}}$ can easily be determined as the smaller of the two distances of both end points of a spherocylinder from the substrate plane. The corresponding function $d_{i j}^{\mathrm{m}}\left(\boldsymbol{r}_{i j}, \widehat{\boldsymbol{u}}_{i}, \widehat{\boldsymbol{u}}_{j}\right)$ is more complex but can be computed numerically using an efficient algorithm proposed by Vega and Lago 29]. Following 21] we model the fluid-substrate interaction via

$$
u_{\mathrm{fs}}=4 \epsilon_{\mathrm{fs}} \rho_{\mathrm{s}}\left[\left(\frac{\sigma}{d_{i k}^{\mathrm{m}}}\right)^{10}-\left(\frac{\sigma}{d_{i k}^{\mathrm{m}}}\right)^{4} g\left(\widehat{\boldsymbol{u}}_{i}\right)\right]
$$

where the parameter $\epsilon_{\mathrm{fs}}$ controls the strength of interaction similar to $\epsilon_{\mathrm{ff}}$ in Eq. (77) and $\rho_{\mathrm{s}}=2 / \ell^{2}$ is the areal density of the substrate where $\ell / \sigma=1 / \sqrt[3]{4}$ is the lattice constant of a single layer of atoms arranged according to the (100) plane of the face-centered cubic lattice. The diameter $\sigma$ of these substrate atoms is taken to be the same as the diameter of a spherocylinder of the confined fluid phase. To obtain Eq. (9) we assume the interaction between the point on the spherocylinder closest to the nearest substrate atom to be described by a conventional Lennard-Jones potential. Holding both the centerof-mass position $\boldsymbol{r}_{i}$ and the orientation $\widehat{\boldsymbol{u}}_{i}$ fixed one then 
averages the interaction with the substrate atom over the entire substrate area. Notice that this procedure gives rise to a prefactor $\frac{2}{5}$ in front of the repulsive term in Eq. (9) which we have deliberately omitted for convenience.

In Eq. (9), $0 \leq g(\widehat{\boldsymbol{u}}) \leq 1$ is the so-called "anchoring function". It permits to discriminate energetically different orientations of a molecule with respect to the substrate plane. In this paper we employ

$$
g(\widehat{\boldsymbol{u}})=\left(\widehat{\boldsymbol{u}} \cdot \widehat{\boldsymbol{e}}_{\mathrm{x}}\right)^{2}+\left(\widehat{\boldsymbol{u}} \cdot \widehat{\boldsymbol{e}}_{\mathrm{y}}\right)^{2}
$$

where $\widehat{\boldsymbol{e}}_{\alpha}$ is a unit vector pointing along the $\alpha$-axis of a space-fixed Cartesian coordinate system. Hence, any molecular arrangement parallel with the substrate plane is energetically favored whereas a homeotropic alignment of a molecule $\left(\widehat{\boldsymbol{u}} \| \widehat{\boldsymbol{e}}_{\mathrm{z}}\right)$ receives an energy penalty by "switching off" the fluid-substrate attraction altogether.

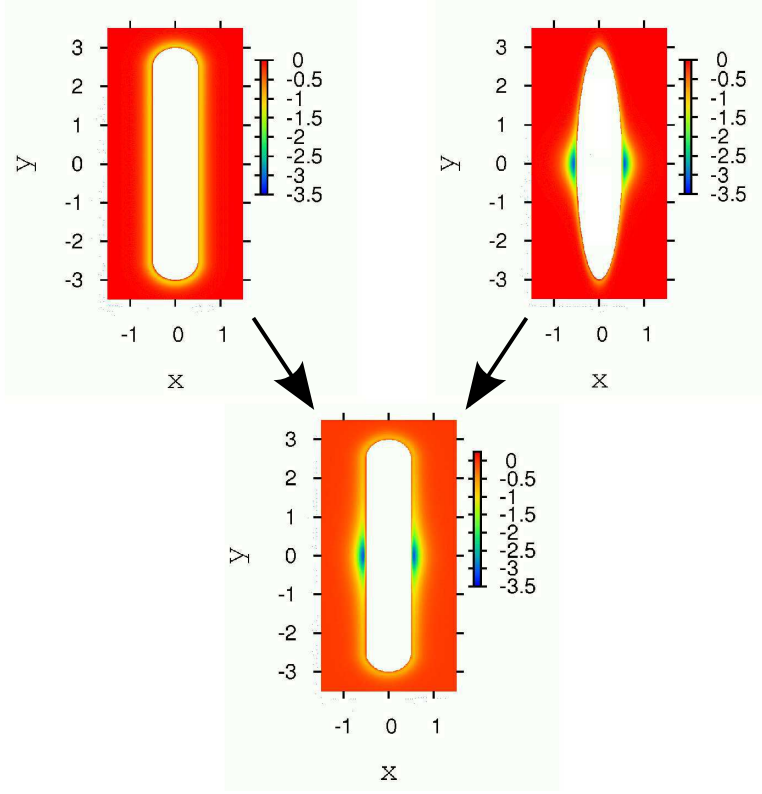

FIG. 3: (Color online) Plots of the equipotential contour plot $u_{\mathrm{ff}} / \epsilon_{\mathrm{ff}}$ for the GBK (bottom), K (top left), and GB (top right) models (see text); color code shown alongside. The area shaded in white is defined through the inequaility $u_{\mathrm{ff}}>0$ therefore approximately representing the molecular shape. In generating the plots we assumed a pair of molecules to be located in the $x-y$ plane with a perfectly parallel orientation (i.e., $\widehat{\boldsymbol{u}}_{i} \cdot \widehat{\boldsymbol{u}}_{j}=1$ ) as a special case (see also Fig. 1). Both $x$ and $y$ are given in units of $\sigma$. Note that the GBK model combines the molecular shape of the $\mathrm{K}$ model with the anisotropy of the GB model.

At this stage a couple of additional comments apply. For example, the reader may notice that Eqs. (7) and (8) are identical with those describing the orientation dependence of the interaction strength in the well-known GayBerne (GB) model of liquid crystals [30]. However, unlike in the GBK model a liquid crystal molecule is perceived as an ellipsoid of revolution in the GB model. This is effected by replacing the function $d_{i j}^{\mathrm{m}}\left(\boldsymbol{r}_{i j}, \widehat{\boldsymbol{u}}_{i}, \widehat{\boldsymbol{u}}_{j}\right)$ in Eq. (6) by one that accounts properly for the shape of a molecule in the GB model [see, for example, Eq. (4) of Ref. 31]. The GBK model is also closely related to the so-called Kihara (K) model 32] of a liquid crystal 33. In this latter model a molecule is again perceived as a spherocylinder but the interaction strength between a pair of molecules depends only on the minimum distance but not on the relative orientation of the molecules of a pair. This is achieved by setting $\kappa^{\prime}=1$ in Eq. (8b) such that $\varepsilon_{\mathrm{ff}}\left(\widehat{\boldsymbol{r}}_{i j}, \widehat{\boldsymbol{u}}_{i}, \widehat{\boldsymbol{u}}_{j}\right)=\epsilon_{\mathrm{ff}}$ in Eq. (7). The shapes of molecules in the K, GB, and GBK models resulting from these manipulations are illustrated by the contour plots in Fig. 3 .

\section{B. Numerical details}

The simulations (both MC and MD) to be presented below are based upon systems containing $N=1500$ molecules. Quantities of interest will be expressed in customary dimensionless (i.e., "reduced") units. For example, length will be expressed in units of $\sigma$, energy in units of $\epsilon_{\mathrm{ff}}$, temperature in units of $\epsilon_{\mathrm{ff}} / k_{\mathrm{B}}$, time in units of $\left(\sigma^{2} m / \epsilon_{\mathrm{ff}}\right)^{1 / 2}$ using $m=1$, and pressure $P_{\|}$in units of $k_{\mathrm{B}} T / \sigma^{3}$ where $P_{\|}=\frac{1}{2}\left(P_{\mathrm{xx}}+P_{\mathrm{yy}}\right)$ is related to diagonal components of the pressure tensor $\mathbf{P}$ acting in the $x-y$ plane.

We employ $\mathrm{MC}$ simulations in an isothermal-isobaric ensemble where we use $N, T, P_{\|}$, and $s_{\mathrm{z} 0}$ as input parameters. In all the simulations we fix $s_{\mathrm{z} 0}=19$ and consider $T=4.0$ and 6.0 . To reduce the computational cost interactions between fluid molecules are cut off beyond a minimum distance $d_{\mathrm{c}}^{\mathrm{m}}=3$. In addition, we employ a conventional Verlet neighborlist where molecules up to a minimum distance of 8 are included as neighbors [34]. In the $x$ - and $y$-directions periodic boundary conditions are applied.

In MC we employ the algorithm of Schoen 35] with one important modification. Rather than changing the area $A$ of the simulation box in the sense of a similarity transformation we allow the individual side lengths $s_{\alpha}$ $(\alpha=x, y)$ to vary independently such that the shape of the simulation cell may change during the course of a simulation. This is important to preserve the in-plane isotropy of $\mathbf{P}$ especially in highly ordered $\mathrm{N}, \operatorname{smA}$, or RN phases. Our runs start from a sufficiently low pressure in the isotropic phase. In the initial configuration molecules are perfectly aligned and then allowed to relax to the isotropic state. The last configuration of this run is then used as a starting configuration of the next run at a slightly higher $P_{\|}$. We continue with such a compression sequence until the last state point of the highest pressure of interest has been reached. We refer to a MC step as $N$ attempted displacements or rotations and one attempted change of $s_{\alpha}$. Displacements and rotations as well as changes for $\alpha=x$ or $\alpha=y$ are performed with equal probability. Our runs are based upon $3 \times 10^{5}$ 
equilibration steps followed by $2 \times 10^{5}$ production steps for thermodynamic states sufficiently far away from any phase transition; around these transitions the number of production steps was enlarged to $7 \times 10^{5}$. However, in changing $A$ as described care has to be taken that none of the instantaneous side lengths $s_{\alpha}$ becomes shorter than twice the neighbor-list cutoff given above.

MC simulations are mainly employed to generate suitable starting configurations for the subsequent MD simulations. In addition, MC is used to independently verify the correctness of the MD simulations through a comparison of equilibrium properties accessible to both types of simulations. In MD we employ the velocity Verlet algorithm for linear molecules in the implementation suggested by Ilnytskyi and Wilson [36]. The iterative solution of the equations of motion is initiated by using the last configuration of the MC simulation at the same state point as a starting configuration where velocities are taken at random from a Maxwell-Boltzmann distribution at the desired temperature. This starting configuration is equilibrated for another $10^{6}$ time steps of $\delta t=0.02$ using a simple velocity rescaling thermostat. The equilibrated system is then monitored for another $2 \times 10^{7}$ time steps with no applied thermostat. During this part of the run the integration time step is reduced to $\delta t=10^{-4}$.

\section{RESULTS}

\section{A. Structure of ordered liquid-crystalline phases}

To characterize the structure of $\mathrm{N}$, smA, and $\mathrm{RN}$ phases we begin by introducing suitably defined order parameters. For nematic phases this is accomplished via the so-called alignment tensor defined as 37]

$$
\mathbf{Q} \equiv \frac{1}{2 N} \sum_{i=1}^{N}\left(3 \widehat{\boldsymbol{u}}_{i} \otimes \widehat{\boldsymbol{u}}_{i}-\mathbf{1}\right)
$$

where " $\otimes$ " denotes the direct (i.e., dyadic) product and $\mathbf{1}$ is the unit tensor. Hence, $\mathbf{Q}$ is a real, symmetric, and traceless second-rank tensor which can be represented by a $3 \times 3$ matrix. For a given configuration $(\boldsymbol{R}, \widehat{\boldsymbol{U}}), \mathbf{Q}$ can be diagonalized in the basis of its three eigenvectors $\widehat{\boldsymbol{n}}_{-}$, $\widehat{\boldsymbol{n}}_{0}$, and $\widehat{\boldsymbol{n}}_{+}$with associated eigenvalues $\lambda_{-}, \lambda_{0}$, and $\lambda_{+}$. Conventionally [38], one then defines the Maier-Saupe [39, 40] nematic order parameter $S=\left\langle\lambda_{+}\right\rangle$where angular brackets denote an ensemble (MC) or time average (MD). On account of its definition, $S=0$ in the isotropic phase if the orientation of the molecules is perfectly random whereas $S=1$ if the molecules are perfectly aligned with the director $\widehat{\boldsymbol{n}}=\widehat{\boldsymbol{n}}_{+}$in the nematic phase.

Even though the nematic phase is characterized by a substantial degree of orientational order the distribution of center-of-mass positions exhibits the typical shortrange order characteristic of ordinary fluids where the typical correlation lengths are comparable to the range of intermolecular interactions. This changes in the smA

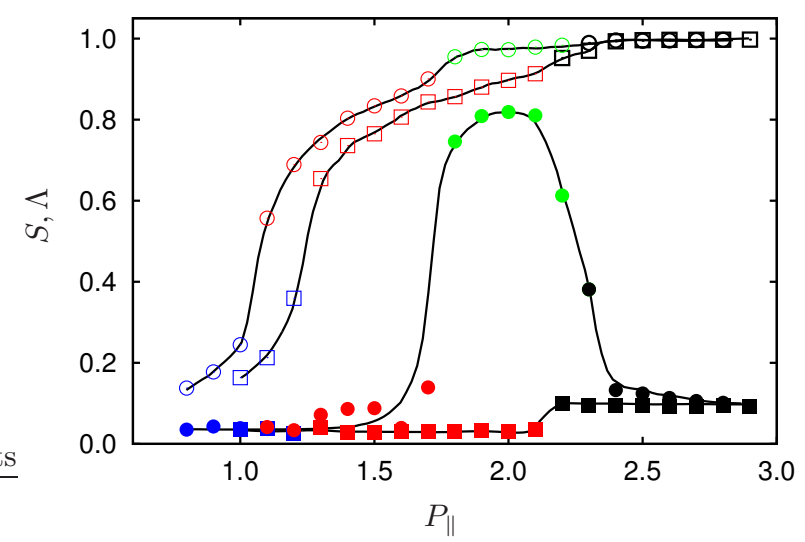

FIG. 4: (Color online) Plots of nematic $S$ (open symbols) and smectic order parameter $\Lambda$ (filled symbols) as functions of pressure $P_{\|}$for various liquid-crystalline phases, $T=4.0$ (circles), and $T=6.0$ (squares). I (blue symbols), $\mathrm{N}$ (red symbols), smA (green symbols), and RN phase (black symbols). The same color code for various liquid-crystalline phases is used throughout this work.

phase where molecules do not only align with $\widehat{\boldsymbol{n}}$ but develop long-range positional order, that is they form individual layers in the direction of $\widehat{\boldsymbol{n}}$. However, positional order remains short-range in directions orthogonal to $\widehat{n}$ such that within a given layer the structure remains fluidlike. Because of the layering of the fluid in the direction of $\widehat{\boldsymbol{n}}$ a suitable quantitative measure of smectic order is given by the leading coefficient of the Fourier transform of the local density $\rho(\boldsymbol{r} \cdot \widehat{\boldsymbol{n}})$

$$
\Lambda \equiv \frac{1}{N}\left\langle\left|\sum_{i=1}^{N} \exp \left[\frac{2 \pi i\left(\boldsymbol{r}_{i} \cdot \widehat{\boldsymbol{n}}\right)}{d}\right]\right|\right\rangle
$$

where $d$ is the spacing between adjacent smA layers. If these layers were ideal, $d=\kappa$ (see Sec. IA ). However, in practice $d \gtrsim \kappa$ on account of thermal fluctuations. Hence, in each configuration $(\boldsymbol{R}, \widehat{\boldsymbol{U}}), d$ is adjusted such as to maximize $\Lambda$. From Eq. (12) it is also apparent that $\Lambda \in[0,1]$. In the nematic phase the superposition of the complex exponential functions in Eq. (12) causes $\Lambda \approx 0$ because in the direction of $\widehat{\boldsymbol{n}}$ a periodic structural feature of peridodicity $d$ does not exist; by a similar token $\Lambda \approx 1$ in the smA phase.

Plots of both $S$ and $\Lambda$ are presented in Fig. 4 for temperatures $T=4.0$ and 6.0. As one can see from the plots, both $S$ and $\Lambda$ are small at low pressures indicating the presence of the I phase. Ideally, $S=0$ in the I phase but in practice attains small positive values of about 0.2 . This is partly due to the substrates which induce a certain degree of nematic order in those portions of the confined liquid crystal that are closest to either substrate. However, a nonvanishing value of $S$ in the isotropic phase is also due to a small but significant finite-size effect that is well-known and has been studied 
quantitatively in the literature [38, 41]. Here we adopt a value $S \simeq 0.4$ as a sensible heuristic definition of the threshold above which the $\mathrm{N}$ phase exists in agreement with the mean-field theory of Maier and Saupe [39, 40]. From Fig. 4 it therefore appears that the $\mathrm{N}$ phase forms somewhere above $P_{\|} \simeq 1.0(T=4.0)$ and $1.2(T=6.0)$, respectively.

Focusing on the lower temperature first, $S$ increases in the $\mathrm{N}$ phase with pressure up to $P_{\|} \simeq 1.7$ indicating the increase of nematic order. Over the same pressure range there are no smectic layers because $\Lambda \lesssim 0.2$ remains relatively small. However, at $P_{\|} \simeq 1.8$, smectic layers are forming as reflected by $\Lambda \simeq 0.8$. Simultaneously, $S$ keeps increasing as well because in the smA phase molecules align themselves even better with $\widehat{\boldsymbol{n}}$. The smA phase remains stable until $P_{\|} \simeq 2.2$ has been attained. Beyond this pressure $S$ remains nearly constant at a high value of about 1.0 whereas $\Lambda$ drops rapidly until a residual small value of about $\Lambda \simeq 0.1$ is assumed which remains constant at all higher pressures considered. The drop in $\Lambda$ with $P_{\|}$clearly reflects the disappearance of smectic layers present at lower $P_{\|}$. Because $S \simeq 1.0$ over the same pressure range we conclude that at sufficiently high pressures the structure of the confined fluid becomes nematic again in a reentrant fashion. To distinguish the highpressure from the low-pressure nematic we refer to the former as $\mathrm{RN}$ because $\mathrm{N}$ and $\mathrm{RN}$ phases are seperated by an intermittent smA phase.

At $T=6.0$ these general features of the orderparameter plots in Fig. 4prevail except that $\Lambda$ never rises above the residual value of about 0.1. Hence, the smA phase does not form at this temperature. Nevertheless, we notice a small, step-like increase at $P_{\|} \simeq 2.2$ in the plot of $\Lambda$. At this and all larger pressures considered the nematic order is high and increases even further reflected by a monotonic increase of $S$ toward its limiting value 1.0. For $P_{\|} \gtrsim 2.2$ the confined fluid exhibits structural features of the RN phase (see below). We shall therefore keep the acronym despite the missing intermittent smA phase.

To get a better feel for the structure of the confined fluid in the N, smA, and RN phases we present configuration "snapshots" from our simulations in Fig. 河 The plot in Fig. [5(a) illustrates the structure of the N phase: one clearly recognizes a preferred net orientation of the molecules but no significant positional order of the center-of-mass distribution. In Fig. 5(b) the layer structure characteristic of the smA phase is visible. These layers disappear in the RN phase as shown in Fig. 5 (c). However, a large degree of orientational order is preserved as one realizes from this latter plot.

A more quantitative analysis of the structure of confined liquid crystals in the various ordered phases is enabled by plots of the local density

$$
\rho(z)=\left\langle\sum_{i=1}^{N} \delta\left(z-z_{i}\right)\right\rangle=\frac{1}{\delta_{\mathrm{z}}}\left\langle\frac{N(z)}{A}\right\rangle
$$
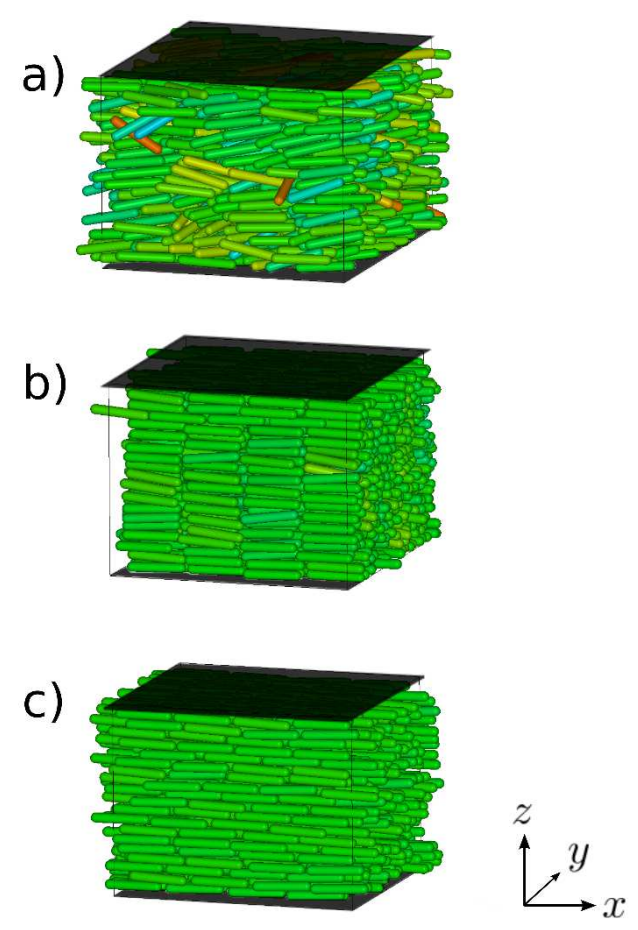

FIG. 5: (Color online) "Snapshots" of characteristic configurations at $T=4.0$; (a) $P_{\|}=1.6(\mathrm{~N})$, (b) $P_{\|}=2.1(\mathrm{smA})$, (c) $P_{\|}=2.9(\mathrm{RN})$ (see Fig. 4). Molecules in green are aligned with $\widehat{\boldsymbol{n}}$ whereas rectangular areas in dark green represent the solid substrates.

where $\delta\left(z-z_{i}\right)$ denotes the Dirac $\delta$-function, $N(z)$ is the number of molecules with their centers of mass located within a interval of width $\delta_{\mathrm{z}}=0.1$, and $A=s_{\mathrm{x}} s_{\mathrm{y}}$ is the instantaneous area of the simulation cell of side length $s_{\alpha}(\alpha=\mathrm{x}, \mathrm{y})$ in the $\alpha$-direction. Hence, $\rho(z)$ is a measure of the probability of finding the center of mass at a specific position $z$. Plots in Fig. [6 illustrate the local structure of the confined liquid crystal in the direction of the substrate normal (i.e., perpendicular to the director $\widehat{\boldsymbol{n}}$ ). As is well-known from simple fluids 42], in confinement $\rho(z)$ is an oscillatory function of position sufficiently close to the substrate surfaces. The oscillations indicate that molecules arrange their centers of mass in individual layers parallel with the substrate. The location of a molecular layer is therefore indicated by a peak in $\rho(z)$. In the immediate vicinity of the solid substrate $\rho(z) \rightarrow 0$ because of the diverging fluid-substrate repulsion as $|z| \rightarrow s_{\mathrm{z}} / 2$.

These general features may be seen from the plots in Fig. 6. One notices that in the $\mathrm{N}$ phase oscillations in $\rho(z)$ are restriczed to the immediate vicinity of the solid substrates [see Fig. [6(a)]. The oscillations decay exponentially as $|z| \rightarrow 0$ with a correlation length determined by bulk properties [43] whereas the spacing 


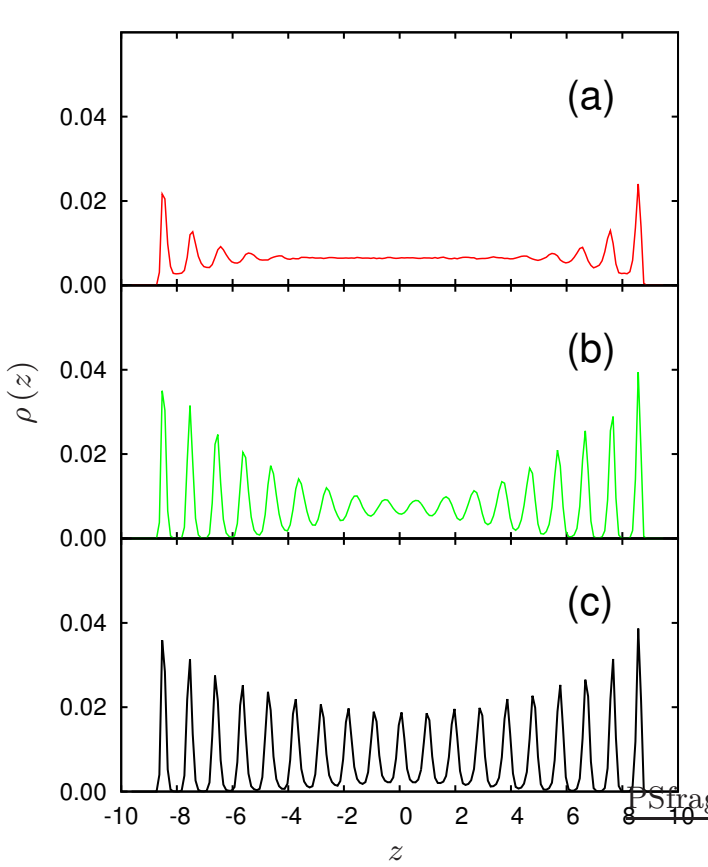

FIG. 6: (Color online) Local density $\rho(z)$ as a function of $z$ for $T=4.0$; (a) $P_{\|}=1.7$, (b) $P_{\|}=2.2$, (c) $P_{\|}=2.3$ (see Fig. 4 for color code).

between neighboring layers reflects the molecular shape [44]. In the $\mathrm{N}$ phase a bulk-like region of considerable width exists in which $\rho(z)$ is constant as the plot in Fig. 6(a) reveals. On the contrary, layering is much more pronounced in the smA phase as a comparison of plots in Figs. 6(a) and 6(b) indicates: Peaks in Fig. 6(b) are taller and because a bulk-like region is absent the entire confined fluid is organized in layers. However, the layered structure becomes weaker as one approaches the center of the slit pore as reflected by the decay of $\rho(z)$ in Fig. 6(b). Finally, in the RN phase layering is even more pronounced as the plot in Fig. 6(c) shows. Moreover, plots in Fig. 6(b) and 6(c) reveal another subtle structural change. Counting maxima in the plots of $\rho(z)$ one realizes that one additional layer of molecules is present in the RN compared with the smA phase. The presence of this additional layer points to a more efficient packing of molecules as far as the arrangement of their centers of mass is concerned. A more efficient packing becomes possible because the layered structure characteristic of the smA phase [see Fig. [5(b)] is lost in the RN phase [see Fig. [5(c)].

Next, at $T=6$ one realizes from Fig. 7 a similar formation of an additional molecular layer but without occurrence of an intermittent smectic phase. Plots in Figs. 7(a) show that for two state points in the nematic phase an increase in pressure only causes the layers already present to become more distinct. For example, $\rho(z)$ drops to zero in regions between individual layers

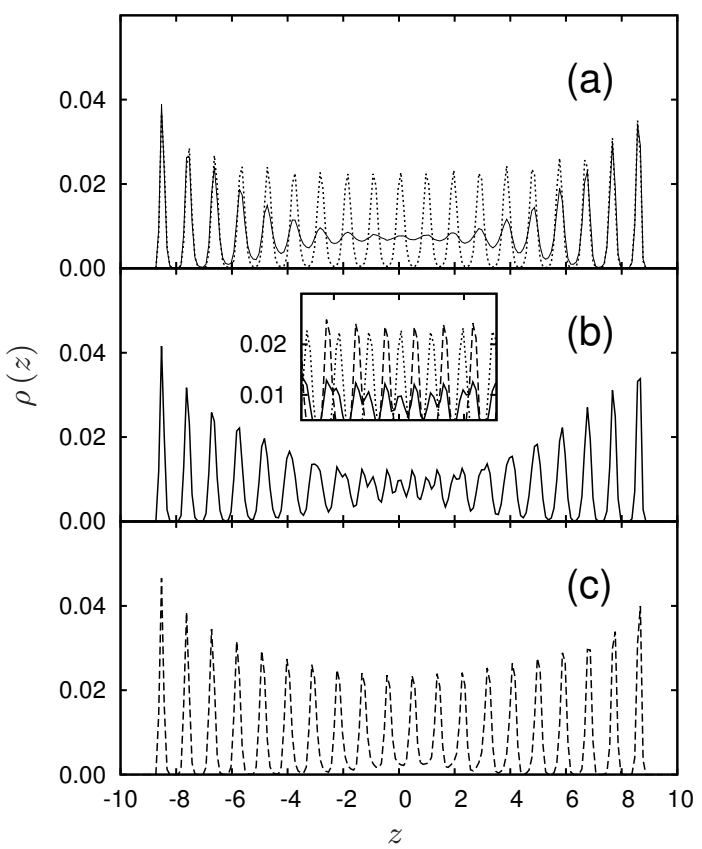

FIG. 7: As Fig. 6 but for $T=6.0$; (a) $P_{\|}=2.3$ (—) and 2.4 (- - -), (b) $P_{\|}=2.5$, (c) $P_{\|}=2.6$ (see Fig. 4 for color code). Note that all parts of the figure refer to $\mathrm{RN}$ states. Inset is a magnification of curves for $P_{\|}=2.4,2.5$, and 2.6 around $z=0$.

at the higher pressure whereas $\rho(z)$ remains nonzero over the corresponding ranges at the lower pressure. The plot in Fig. 7(b) corresponds to a state point where the confined fluid undergoes the subtle reorganization that will eventually lead to the growth of an additional layer. As one approaches the midplane located at $z=0$ individual peaks in $\rho(z)$ first broaden and eventually split up into two unresolved peaks. At an even higher pressure considered in Fig. 7(c) we realize that the fluid has completed its structural reorganization and a new fluid layer emerges as we can see by comparing this plot with its counterparts shown in Fig. 7(a). Moreover, visual inspection of a characteristic configuration [see Fig. [5(c)] reveals that in the $\mathrm{RN}$ phase molecules appear in stringlike arrangements.

In our terminology a "string" is a sequence of molecules arranged in space like individual cars of a train with their long axes all pointing in the same direction. The "snapshot" of a typical configuration presented in Fig. [5(c) already indicates that these supramolecular strings are omnipresent in the $\mathrm{RN}$ phase. In mathematical terms an ideal string can therefore be defined through the relations

$$
\begin{aligned}
\widehat{\boldsymbol{u}}_{i} \cdot \widehat{\boldsymbol{u}}_{j} & =1 \\
\widehat{\boldsymbol{u}}_{i} \cdot \widehat{\boldsymbol{r}}_{i j} & =\widehat{\boldsymbol{u}}_{j} \cdot \widehat{\boldsymbol{r}}_{i j}=1
\end{aligned}
$$

where, of course, one of the two scalar products in 
a)

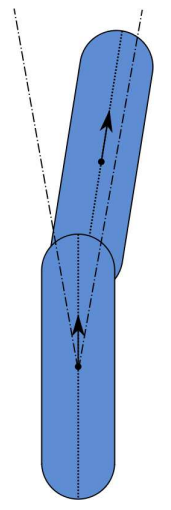

b)

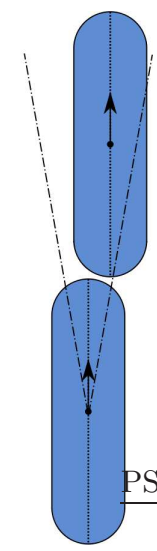

FIG. 8: (Color online) Sketch of a pair of spherocylinders (see Fig. 11). (a) Shifted side-side conformation, (b) As (a), but for a string-like conformation. The wedge-shaped areas corresponds to the solid angle $\widehat{\boldsymbol{u}}_{i} \cdot \widehat{\boldsymbol{u}}_{j} \geq \delta_{\cos \theta}$ used in Eq. (16) which we exaggerate for illustrative purposes.

Eq. 14b) is redundant. In practice, however, small deviations from perfect alignment of molecules in a string arise on account of thermal fluctuations. A good measure of the extent to which a pair of molecules is arranged in a string-like conformation approximately may be defined by decomposing the distance vector $\boldsymbol{r}_{i j}$ between the centers of mass of nearest-neighbor molecules $i$ and $j$ into components parallel and perpendicular to $\widehat{\boldsymbol{u}}_{i}$ through the expressions (see Fig. 1)

$$
\begin{aligned}
\boldsymbol{r}_{i j}^{\|} & =\widehat{\boldsymbol{u}}_{i} \cdot \boldsymbol{r}_{i j} \\
\boldsymbol{r}_{i j}^{\perp} & =\boldsymbol{r}_{i j}-\left(\widehat{\boldsymbol{u}}_{i} \cdot \boldsymbol{r}_{i j}\right) \widehat{\boldsymbol{u}}_{i}
\end{aligned}
$$

For a string-like configuration of a pair of molecules one anticipates $r_{i j}^{\perp} \equiv\left|\boldsymbol{r}_{i j}\right| \rightarrow 0$. However, a second characteristic conformation characterized by relatively small values of $r_{i j}^{\perp}$ is conceivable. In this conformation molecules are arranged in a shifted side-side arrangement [see Fig. 8(a)]. Plots of configurational snapshots in Fig. 5 clearly show that also unshifted side-side arrangements exist both in the $\mathrm{N}$ and in the $\mathrm{RN}$ phase. This is because they are energetically favored according to Fig. 3(a). However, they are also present to some extent in other phases as snapshots in Figs. 5(a) and 5 (c) indicate. To eliminate these unwanted unshifted side-side conformations in favor of only shifted ones it is prudent from an operational point of view to consider only the subset $\left\{\widetilde{\boldsymbol{r}}_{i j}^{\perp}\right\} \subseteq\left\{\boldsymbol{r}_{i j}^{\perp}\right\}$ defined through

$$
\begin{aligned}
\left\{\widetilde{\boldsymbol{r}}_{i j}^{\perp}\right\} \equiv & \left\{\boldsymbol{r}_{i j} \mid r_{i j} \leq r_{\mathrm{c}}^{\perp}=\kappa+\delta_{\kappa} \wedge r_{i j}^{\|} \geq \frac{\kappa}{2}+1\right. \\
& \left.\wedge \widehat{\boldsymbol{u}}_{i} \cdot \widehat{\boldsymbol{u}}_{j} \geq \delta_{\cos \theta}\right\}
\end{aligned}
$$

where we arbitrarily take $\delta_{\kappa}=0.2$ and $\delta_{\cos \theta}=0.98$. To see that this rather complex definition selects only those conformations sketched in Figs. 8(a) and 8(b) one needs to realize that the first of the three conditions in
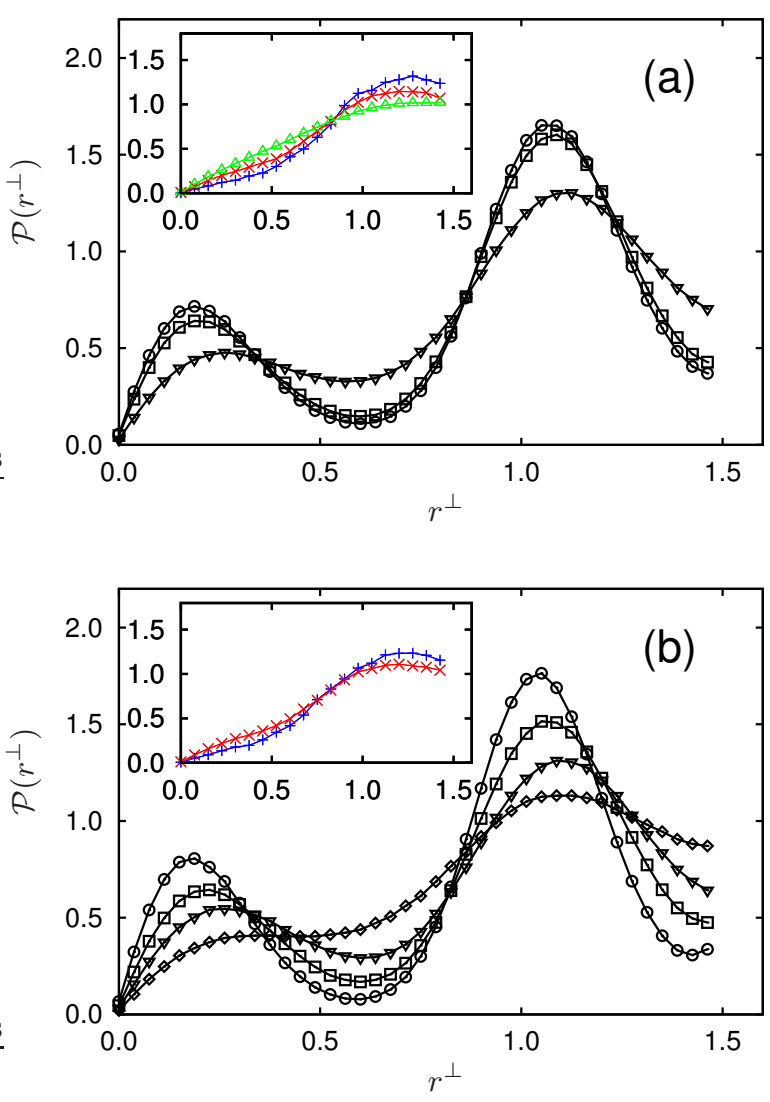

FIG. 9: (Color online) Probability distribution $\mathcal{P}$ of $r^{\perp}$ (see Figs. 1 and 8 Eqs. (15)-(17)]. (a) $T=4.0$ and $P_{\|}=2.3$ $(\nabla), P_{\|}=2.6(\square)$, and $P_{\|}=2.8(\mathrm{O})$; inset: $P_{\|}=0.9(+)$, $P_{\|}=1.4(\times)$, and $P_{\|}=1.9(\triangle)$. (b) $T=6.0$ and $P_{\|}=2.2$ $(\diamond), P_{\|}=2.4(\nabla), P_{\|}=2.5(\square)$, and $P_{\|}=2.9(\mathrm{O})$; inset: $P_{\|}=1.1(+)$ and $P_{\|}=1.7(\times)$. For line and symbol color code see Fig. 4

Eq. (16) selects nearest neighbors $\{j\}$ of a given molecule $i$, the second condition favors head-tail-like conformations, whereas the third one selects molecules with nearly perfectly aligned long molecular axes. It is then convenient to introduce the probability

$$
\mathcal{P}\left(r^{\perp}\right)=\frac{\mathcal{P}_{0}}{N}\left\langle\sum_{i=1}^{N} \sum_{j=1 \neq i}^{N} \delta\left(r^{\perp}-\widetilde{r}_{i j}^{\perp}\right)\right\rangle
$$

for a quantitative discussion of the formation of stringlike structures where $\mathcal{P}_{0}$ is a normalization constant which we determine via

$$
\int_{0}^{r_{c}^{\perp}} \mathrm{d} r^{\perp} \mathcal{P}\left(r^{\perp}\right) \stackrel{!}{=} 1
$$

In the actual simulations we obtain $\mathcal{P}\left(r^{\perp}\right)$ as a histogram using $\delta r^{\perp}=0.02$ as the bin width. 
Plots in Fig. 9 show $\mathcal{P}\left(r^{\perp}\right)$ for different temperatures and pressures in the I, N, smA, and RN phase. At sufficiently low pressures it is evident from the insets in Figs. 9(a) and 9(b) that $\mathcal{P}\left(r^{\perp}\right)$ increases monotonically with $r^{\perp}$ and reaches a broad maximum located approximately at $r^{\perp} \simeq 1.25$ irrespective of whether the I, N, or smA phase is considered. That $\mathcal{P}\left(r^{\perp}\right)$ rises with $r^{\perp}$ may be ascribed to molecules in the I, N, and smA phase that arrange their long axes locally to a certain degree. This local order is solely caused by the intermolecular interaction potential which favors local side-side conformations of molecules [see Fig. [3(a)].

In the RN phase the form of $\mathcal{P}\left(r^{\perp}\right)$ is completely different. Here the plots in Fig. 9(a) and 9(b) exhibit two maxima. The largest one slightly above $r^{\perp} \simeq 1.0$ corresponds to conformations in which neighboring particles are arranged in the shifted side-side conformation depicted schematically in Fig. 8(a). Note that these values of $r^{\perp}$ are slightly below the position of the minimum of the interaction potential (see Fig. 21). The secondary maximum of $\mathcal{P}\left(r^{\perp}\right)$ in the $\mathrm{RN}$ phase at $r^{\perp}<0.5$ corresponds to string-like conformations schematically illustrated by the plot in Fig. 8(b). Were the string-like conformation perfect one would anticipate a peak of $\mathcal{P}\left(r^{\perp}\right)$ at $r^{\perp}=0$. However, on account of thermal fluctuations these ideal string-like conformations are only infrequently observed. For example, closer scrutiny would reveal that $\mathcal{P}\left(r^{\perp}\right) \neq 0$ at $r^{\perp}=0$ indicating that a very small fraction of molecules does, in fact, assume an ideal stringlike conformation. As $P_{\|}$increases one notices that both peaks of $\mathcal{P}\left(r^{\perp}\right)$ shift to progressively smaller $r^{\perp}$. This fact reflects an arrangement of molecules in more perfect string-like conformations with increasing pressure as one would expect intuitively.

One also notices from plots in Figs. 9(a) and 9(b) that the curves seem to intersect in isolated points at characteristic values of $r^{\perp}$. These intersections may be interpreted as "isosbestic" points (derived from the Greek words isos: equal, the same and sbestos: extinguishable) [45], a concept widely known in spectroscopy. The occurrence of isosbestic points is relatively simple to grasp. We assume that $\mathcal{P}\left(r^{\perp}\right)$ can be decomposed into a sum of three contributions:

1. a probability distribution centered at $r^{\perp} \approx 1.0$ corresponding to a population of shifted side-side molecular conformations [see Fig. 8(a)],

2. a probability distribution centered at $r^{\perp} \approx 0.25$ corresponding to a population of shifted string-like molecular conformations [see Fig. 8(b)],

3. and a probability distribution representing all other molecular conformations satisfying Eq. (16) and embracing the entire range of $r^{\perp}$.

For sufficiently large $P_{\|}$the distributions of 1 and 2 can be assumed to be well separated whereas 3 may be thought of as a broad background distribution. Intersections between distributions 1 and 3 or between 2 and
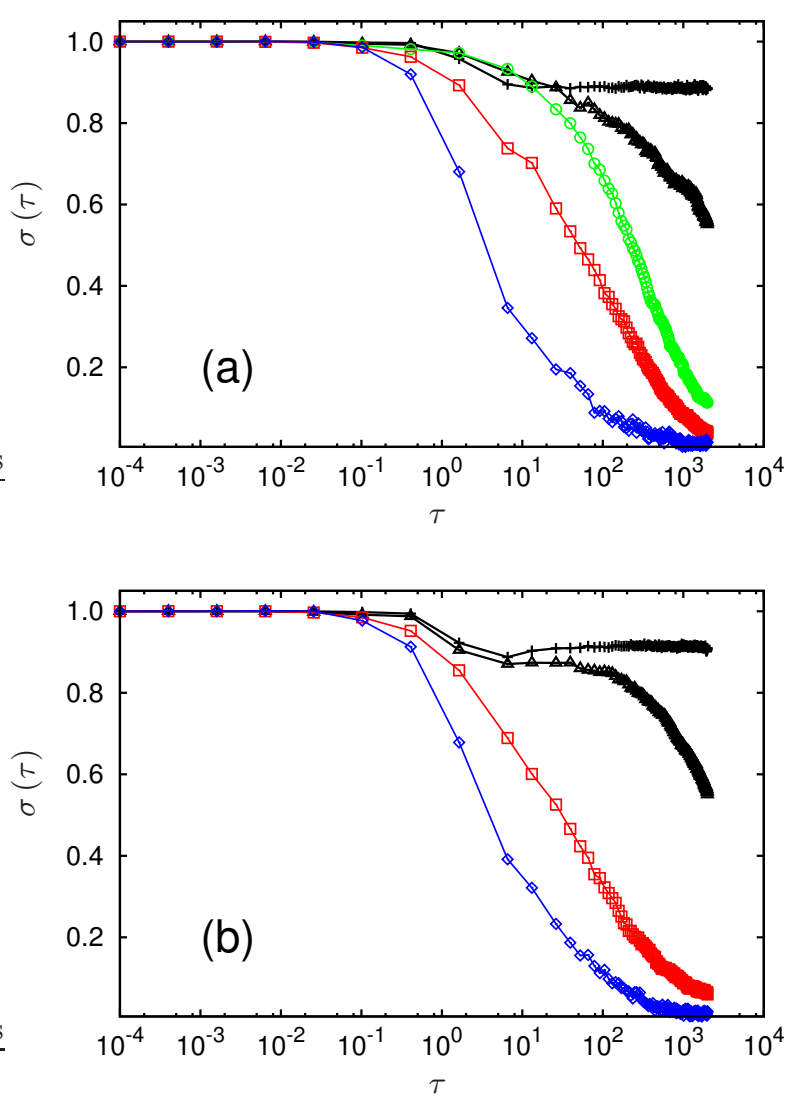

FIG. 10: (Color online) (a) Persistence probability $\sigma(\tau)$ as a function of time $\tau$ and $T=4.0 ; P_{\|}=0.9(\diamond), P_{\|}=1.7(\square)$, $\left.P_{\|}=2.1(\mathrm{O}), P_{\|}=2.3\right)(\mathbf{\Lambda}), P_{\|}=2.8(+)$. (b) as (a), but for $T=6.0$ and $P_{\|}=1.1(\diamond), P_{\|}=1.9(\square), P_{\|}=2.4(\boldsymbol{\Delta})$, and $P_{\|}=2.9(+)$ (see Fig. 4 for color code).

3. respectively, will give rise to isosbestic points. As $P_{\|}$ increases distributions 1 and 2 become sharper and increase in height at the expense of the background distribution 3 furthermore, maxima of both distributions 1 and 2 shift to lower values of $r^{\perp}$ indicating an increase in order. Importantly, the emergence of two well-defined peaks in $\mathcal{P}\left(r^{\perp}\right)$ is a fingerprint of the $\mathrm{RN}$ phase and could therefore be taken as computational evidence to detect $\mathrm{RN}$ phases even though its experimental significance may be limited. Incidentally, at low $P_{\|}$(i.e., in the $\mathrm{I}, \mathrm{N}$, or smA phase) the only important distributions are 1 and 2 because string-like conformations are statistically irrelevant. Consequently, only one isosbestic point exists as shown in the insets of Fig. 9

\section{B. Self-diffusivity in liquid crystalline phases}

The formation of string-like conformations has important ramifications as far as dynamic properties of the RN phase are concerned. To illustrate this we begin by con- 
sidering a persistence probability

$$
\sigma(\tau) \equiv \frac{\left\langle\sum_{(i, j)} P_{2}\left[\eta_{i j}(t+\tau)\right]\right\rangle_{t}}{\left\langle\sum_{(i, j)} P_{2}\left[\eta_{i j}(t)\right]\right\rangle_{t}}
$$

defined as the correlation function of the angle between a pair of molecules $(i, j)$ which at the initial time $t$ satisfy the conditions for a string-like conformation introduced in Eq. (16). Subscript " $t$ " is attached to the angular brackets to emphasize that averaging involves a sufficiently large number of statistically uncorrelated time origins which implicitly invokes the principle of stationarity of temporal correlation functions in equilibrium systems [46]. In Eq. [19) $\eta_{i j} \equiv \widehat{\boldsymbol{u}}_{i} \cdot \widehat{\boldsymbol{r}}_{i j}$ is the cosine of the angle between the instantaneous orientation of a reference molecule $i$ and the unit vector connecting its center of mass to that of another molecule $j$ as time passes [see Fig. 1. Because of this definition, $\eta_{i j}(t+\tau)$ is a measure of the relative orientation of a molecular pair at time $t+\tau$ given that its orientation at time $t$ is $\eta_{i j}(t)$. Therefore, $\sigma(\tau) \rightarrow 1$ as $\tau \rightarrow 0$ in agreement with plots in Figs. 10(a) and 10(b) for various temperatures and pressures. At sufficiently low $P_{\|}, \sigma(\tau)$ eventually decays with increasing time. The decay is delayed the more as the nature of the liquid-crystalline phase changes in the direction $\mathrm{I} \rightarrow \mathrm{N} \rightarrow \mathrm{smA}$, that is with increasing $P_{\|}$[see Fig. [10(a)].

One also notices from Fig. 10(a) that the decorrelation of relative orientations becomes very slow as soon as one enters the RN phase. This is already evident from the plot for $P_{\|}=2.3$ immediately after the smA-RN phase transition has occurred (see Fig. (4). However, the corresponding plot in Fig. 10(a) shows that $\sigma(\tau)$ eventually decays at long times. This is apparently not so at the higher pressure $P_{\|}=2.8$ in the RN phase. The relevant plot in Fig. 10(a) shows only a weak decay of $\sigma(\tau)$ over an intermediate time range $1 \lesssim \tau \lesssim 10$ and then remains nearly constant for longer times $\tau>10^{3}$. The shape of $\sigma(\tau)$ seems to reflect a two-stage decorrelation process involving those conformations represented by the bimodal distribution $\mathcal{P}\left(r^{\perp}\right)$ plotted in Fig. 9(a). In fact, intuitively it seems sensible to assume that decorrelation during the intermediate time interval is associated with the orientational dynamics of shifted side-side conformations depicted in Fig. 8(a). These conformations are expected to be less stable than the string-like ones depicted in Fig. 8(b). This notion is further corroborated by the slowing down of the dynamics at longer times with increasing $P_{\|}$which causes the population of string-like conformations to increase according to plots in Figs. 9 . This qualitative picture remains unaltered at the higher $T=6.0$ as parallel plots in Fig. 10(b) show. However, at this temperature the smA phase is absent in agreement with our earlier analysis of various structural quantities.

From the discussion of orientational relaxation one may get the impression that the $\mathrm{RN}$ phase is rather
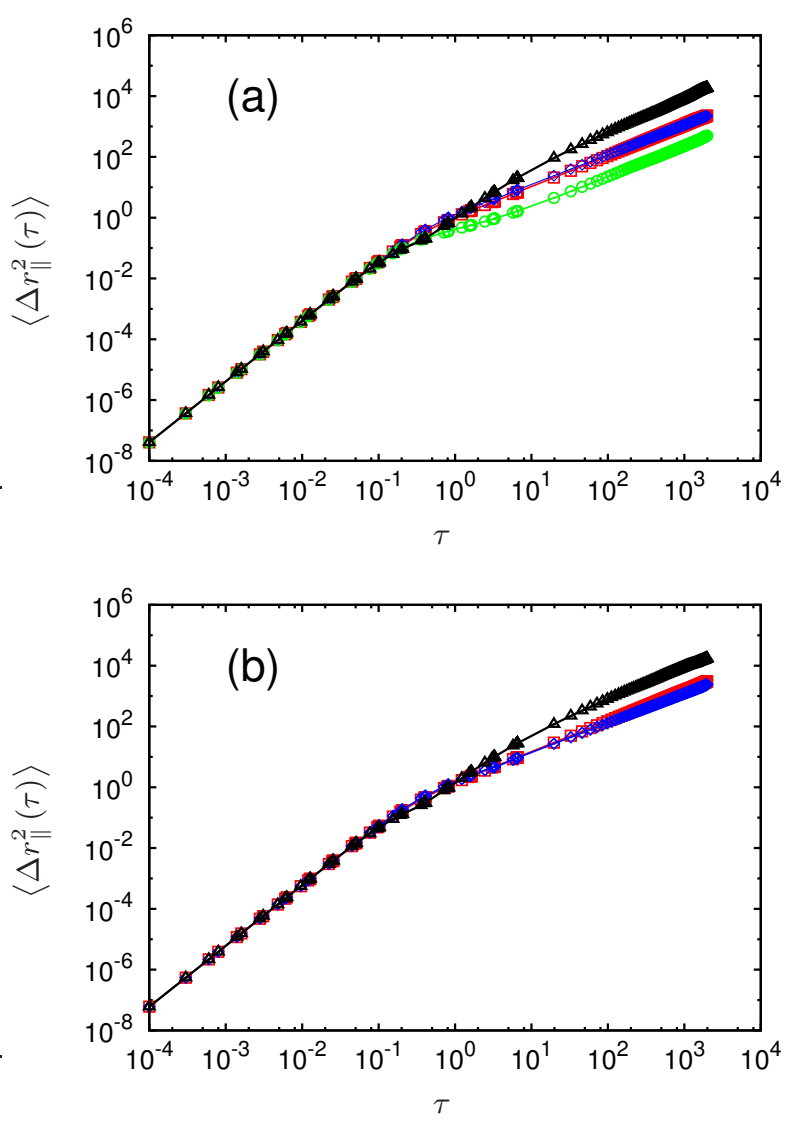

FIG. 11: (Color online) (a) MSDs $\left\langle\Delta r_{\|}^{2}(\tau)\right\rangle$ as functions of time $\tau$ and $T=4.0$ and various liquid-crystalline phases at $P_{\|}=0.9(\mathrm{I})(\diamond), P_{\|}=1.7(\mathrm{~N})(\square), P_{\|}=2.1(\mathrm{smA})(\mathrm{O})$, and $P_{\|}=2.6(\mathrm{RN})(\triangle)$. (b) as (a), but for $T=6.0$ and $P_{\|}=1.1$ $(\mathrm{I})(\diamond), P_{\|}=1.9(\mathrm{~N})(\square)$, and $P_{\|}=2.4(\mathrm{RN})(\triangle)$ (see Fig. 4 for color code).

sluggish with respect to its dynamics. While this is certainly true for the rotational dynamics it turns out that mass transport is actually quite fast in the $\mathrm{RN}$ phase. A suitable measure of mass transport is the mean square displacement (MSD) of molecules. It measures the onaverage net distance a molecule's center of mass is able to travel in a given amount of time. Because of the large degree of nematic order characteristic of the RN phase it seems sensible to introduce a specialized MSD by considering displacements of molecules in the direction of their long axes. Specifically, we define $\boldsymbol{r}_{i}^{\|} \equiv \widehat{\boldsymbol{u}}_{i} \cdot \boldsymbol{r}_{i}$ such that the associated MSD for the displacement of molecules in the direction of their long axes may be cast as

$$
\left\langle\Delta r_{\|}^{2}(\tau)\right\rangle_{t} \equiv \frac{1}{N}\left\langle\sum_{i=1}^{N}\left[\boldsymbol{r}_{i}^{\|}(t+\tau)-\boldsymbol{r}_{i}^{\|}(t)\right]^{2}\right\rangle_{t}
$$

Plots in Fig. 11 illustrate the variation of the MSD with time on a double-logarithmic scale. At short times $\tau \leq 10^{-1}$ MSDs for various phases collapse onto a sin- 


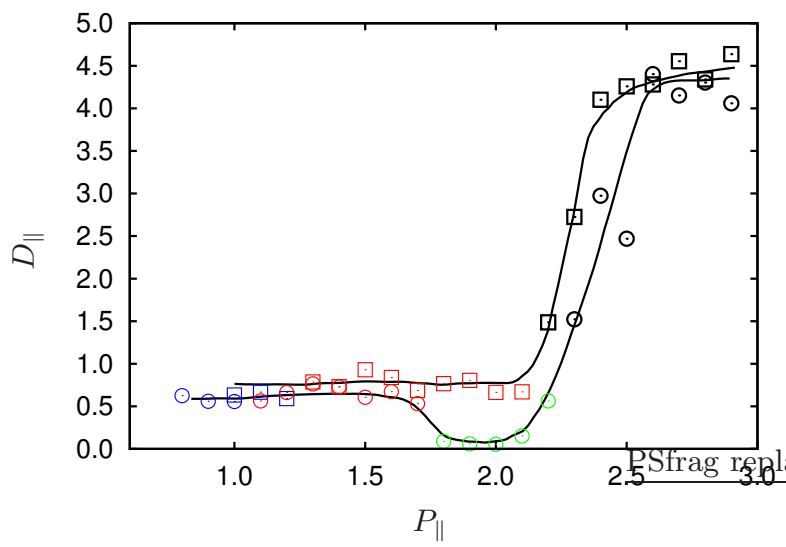

FIG. 12: (Color online) Parallel self-diffusion coefficient $D_{\|}$ as a function of transverse pressure $P_{\|}$. Circles correspond to $T=4.0$ whereas squares refer to $T=6.0$ (see Fig. 4 for color code).

gle curve. In this regime, known as the ballistic regime, the MSD increases proportionally to $\tau^{2}$. In the ballistic regime molecules travel freely, that is for sufficiently short times interactions with neighboring molecules are inconsequential. As a consequence, the specific nature of the phase under study does not matter such that the MSDs for different phases become indistinguishable and can be represented by a unique curve.

At sufficiently long times, however, the motion of molecules becomes diffusive, that is $\left\langle\Delta r_{\|}^{2}(\tau)\right\rangle_{t} \propto \tau$. In the diffusive regime intermolecular interactions do, of course, matter greatly unlike in the ballistic regime. Therefore, in the diffusive regime plots in Figs. 11(a) and 111(b) cannot be represented by a single curve but differ between I, N, smA, and RN phases. An inspection of the MSD for the smA phase reveals that ballistic and diffusive regimes are separated by a third, subdiffusive regime in which $\left\langle\Delta r_{\|}^{2}(\tau)\right\rangle_{t} \propto \tau^{\alpha(\tau)}$ where $1 \lesssim \alpha(\tau) \lesssim 2$ varies continuously. The crossover region of subdiffusive behavior reflects the presence of smectic layers because $\left\langle\Delta r_{\|}^{2}(\tau)\right\rangle_{t}$ is a measure for mass transport in a direction normal to the layer plane [see Fig. 5(b)]. Diffusive motion of molecules out of their original smectic layer into a neighboring one is inhibited by the compactness of the layered structure. However, within each layer there is sufficiently little order such that molecules may eventually move from their original to a neighboring layer. This process demarcates the onset of ordinary diffusive motion. Depending on the local compactness of the smectic layers it may take some molecules longer than others before they exhibit diffusive motion and this is the reason why the subdiffusive regime is characterized by a constant change in the time dependence of the MSD characterized by the exponent $\alpha(\tau)$.

As a quantitative measure of mass transport in the

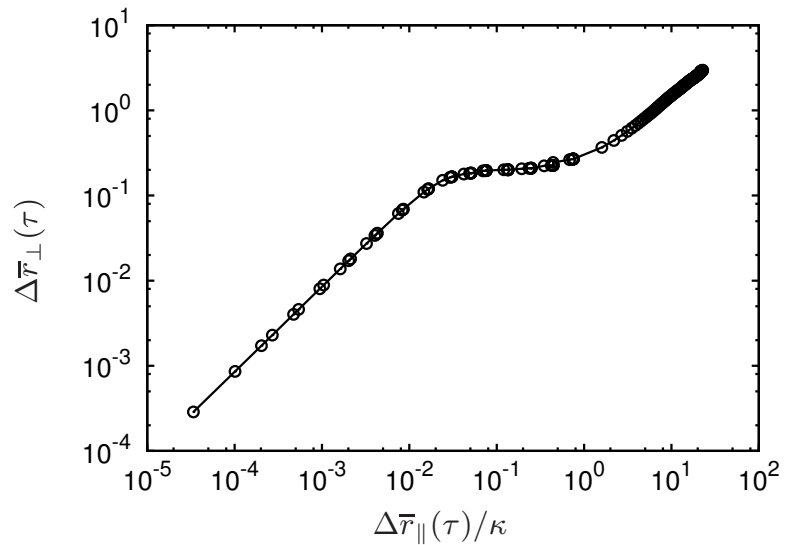

FIG. 13: Root MSD $\Delta \bar{r}_{\perp}(\tau)$ for the average displacement of molecules in a direction perpendicular to their long axes as a function of the root MSD $\Delta \bar{r}_{\|}(\tau)$ for the average displacement of molecules in a direction parallel to these axes in units of the aspect ratio $\kappa$. Data correspond to the RN phase at $T=4.0$ and $P=2.6$. Solid line is a fit to guide the eye.

diffusive regime it is convenient to introduce the selfdiffusion coefficient $D_{\|}$via the expression

$$
D_{\|}=\lim _{\tau \rightarrow \infty} \frac{1}{2 \tau}\left\langle\Delta r_{\|}^{2}(\tau)\right\rangle_{t}
$$

which corresponds to the long-time slope of the MSDs plotted in Figs. 11] From that definition and the plots in Figs. 11 one immediately anticipates a self-diffusion constant which is largest in the RN phase compared with the other three phases. In fact, the plot in Fig. 12 shows a dramatic increase of $D_{\|}$as one enters the RN phase whereas lower-pressure (I, N, or smA) phases exhibit rather small self-diffusivity. In particular, the smA phase is characterized by nearly vanishing self-diffusion constants which can be rationalized as above where we argued that the relatively compact layered structure makes it difficult for molecules to diffuse out of their original layer and penetrate into a neighboring one. The dramatic increase in mass transport in the direction of $\widehat{\boldsymbol{n}}$ in combination with nearly perfect nematic order prompted us to refer to liquid crystals in the RN phase as "supernematics" 21].

More detailed insight into the dynamics of mass transport in the RN phase can be gained by also considering the MSD for molecular displacements in the direction orthogonal to the molecules' long axes [47] to which we refer as $\left\langle\Delta r_{\perp}^{2}(\tau)\right\rangle_{t}$ by analogy with Eq. (20) where, of course, we replace $\boldsymbol{r}_{i}^{\|}$by its counterpart $\boldsymbol{r}_{i}^{\perp} \equiv \boldsymbol{r}_{i}-\left(\widehat{\boldsymbol{u}}_{i} \cdot \boldsymbol{r}_{i}\right) \widehat{\boldsymbol{u}}_{i}$. Plots of the root MSD's for perpendicular versus parallel molecular displacements $\Delta \bar{r}_{\perp, \|} \equiv \sqrt{\left\langle\Delta r_{\perp, \|}^{2}(\tau)\right\rangle_{t}}$ in Fig. 13 reveal that dynamically distinct regimes exist. In the ballistic regime the plot in Fig. 13 increases linearly with slope 1 which can be rationalized as follows. 
Over the relatively short times characteristic of the ballistic regime molecules move freely in space. Hence, their self-diffusion is largely controlled by the free volume surrounding a molecule. In the $\mathrm{RN}$ phase the shape of the free volume should be closely related to the shape of an individual spherocylinder. Therefore, rescaling $\Delta \bar{r}_{\|}(\tau)$ by the aspect ratio $\kappa$ and expressing $\Delta \bar{r}_{\perp}(\tau)$ in units of $\sigma$ one anticipates the plot in Fig. 13 to increase linearly with a slope of 1 in the ballistic regime where both $\Delta \bar{r}_{\perp}(\tau)$ and $\Delta \bar{r}_{\|}(\tau)$ are small compared with $\sigma$. If this condition does not hold any longer intermolecular interactions begin to matter and self-diffusion is no longer ballistic. The plot in Fig. 13 shows that during the initial stage of non-ballistic self-diffusion the plot of $\Delta \bar{r}_{\perp}(\tau)$ versus $\Delta \bar{r}_{\|}(\tau)$ levels off and reaches a plateau. During this stage molecules diffuse in string-like conformations which remain intact, that is the group of molecules forming a string-like conformation stays together as an entity. However, if both $\Delta \bar{r}_{\perp}(\tau)$ and $\Delta \bar{r}_{\|}(\tau)$ are sufficiently larger than $\sigma$ the plot in Fig. 13 increases again linearly but with a somewhat smaller slope of about 0.88 compared with the initial ballistic regime. Because in this second linear regime $\Delta \bar{r}_{\perp}(\tau)$ is of the order of several $\sigma$, diffusive motion must involve the exchange of individual molecular units between neighboring supramolecular "strings" which illustrates the complexity of the dynamics in the supernematic $\mathrm{RN}$ phase.

\section{SUMMARY AND CONCLUSIONS}

In this paper we elucidate the connection between the formation of string-like conformations of elongated molecules in the RN liquid-crystalline phase and enhanced self-diffusivity observed earlier by us [21] in these phases. We employ a combination of MC and MD simulations performed in an isothermal-isobaric and in the microcanonical ensemble, respectively. Under conditions of confinement a sequence of mesophases is observed. These involve the more conventional I and $\mathrm{N}$ phases and, under suitable thermodynamic conditions, a smA phase. At higher pressures (densities) the more exotic RN phase may form which is a nematic phase with surprisingly large nematic order and enhanced self-diffusivity in the direction of the nematic director $\widehat{\boldsymbol{n}}$. Quantitatively, we express the extent of mass transport in terms of the selfdiffusion constant $D_{\|}$. In the RN phase $D_{\|}$may exceed that characteristic of the I, N, and smA phases by up to an order of magnitude.

Our analysis of the underlying structure reveals that the RN phase is characterized by so-called "strings", that is groups of molecules arranged such that their long axes and their center-of-mass distance vectors point in the direction of $\widehat{\boldsymbol{n}}$ with only very little deviation from a perfect arrangement. These arrangements can clearly be seen in representative "snapshots" of individual configurations. On the contrary, string-like conformations are absent as far as I, N, or smA phases are concerned. For the smA phase this may seem surprising at first on account of the formation of distinct layers in the direction of $\widehat{\boldsymbol{n}}$. However, one has to bear in mind that in each individual layer the order of center-of-mass arrangements is still rather low which apparently prevents the formation of strings between adjacent layers. The reader should also note that if one considers a single isolated string that remains intact indefinitely one would anticipate $\left\langle\Delta r_{\|}^{2}(\tau)\right\rangle_{t} \propto \sqrt{\tau}$ characteristic of single-file diffusion [50]. In single-file diffusion the time dependence of the MSD is caused by the fact that particles cannot pass each other, a situation encountered experimentally for the motion of molecules in the narrow spaces existing in materials such as zeolites [51], single-wall carbon nanotubes 52], or colloids confined to one-dimensional channels [53]. However, in the present case one needs to realize that we are confronted with a totally different physical situation. As the "snapshots" of configurations show many strings exist which are misaligned in the direction in which they diffuse. At sufficiently long times individual molecules are exchanged between neighboring supramolecular "string-like" units. As a result, ordinary Fickian diffusion is the consequence in the limit of sufficiently long times.

The interesting question then becomes: why is this diffusive motion enhanced? In fact, based upon plots of the equipotential surface one would assume that even in a string-like conformation molecules would "feel" most comfortable if individual members in both strings are arranged such that neighboring strings are perfectly aligned because of the energetically favored side-side arrangement of pairs of molecules. If this were the case one would expect self-diffusion in the direction of the stringlike conformations to actually be slowed down rather than enhanced on account of restoring forces acting in the direction of $\widehat{\boldsymbol{n}}$ that pull molecules back to relative arrangements corresponding to locations of potential minima. However, this rationale ignores the fact that $\mathrm{RN}$ phases form at relatively high pressures at which individual strings are "squeezed" together in the direction perpendicular to the plane of the solid substrates. That strings are actually squeezed in that direction is reflected by the local density which shows that in the RN phase one additional layer of molecules can be accommodated compared with either $\mathrm{N}$ or smA phases at lower pressures. In other words, molecules in neighboring strings are pushed towards each other. This happens to an extent such that the attractive interactions between any pair of molecules pertaining to different strings (and therefore the restoring forces in the direction of $\widehat{\boldsymbol{n}}$ ) are overcome at least partly. In other words, the average distance $r^{\perp}$ between the molecules of a pair decreases with $P_{\|}$such that the pair slowly "climbs up" the repulsive part of the fluidfluid interaction potential. Thereby, the net attraction between the two molecules is reduced as revealed by the shift of the taller maximum of $\mathcal{P}\left(r^{\perp}\right)$ plotted in Fig. 9] As a result diffusion in string-like conformations is enhanced rather than diminished. 
This mechanism is similar to one proposed for mass transport in zeolites. For example, based upon experiments using quasielastic neutron scattering and corresponding MD simulations, Borah et al. could demonstrate that in NaY zeolites severe confinement causes selfdiffusivity to become maximum under geometrically favorable conditions 22]. This effect known as "levitation" is most pronounced if the narrowest void in the zeolite is comparable to the optimum distance between a diffusing molecule and the pore wall which is, of course, determined by the underlying fluid-wall interaction potential. If the width of the void is either too small or too large, self-diffusivity is lower than under the optimum confinement conditions. Levitation is quite regularly observed in zeolites of various kinds (see Ref. 22 and references therein).

At this point one may ask: Is there any experimental evidence supporting the enhanced self-diffusion that we observe in our simulations? Though the experimental data so far communicated in the literature do not yet provide a rigorous confirmation of our theoretical predictions, some of these data are in reasonable qualitative agreement with our simulations. For example, extrapolating longitudinal relaxation rates reported in Ref. 25 from the $\mathrm{RN}$ to the $\mathrm{N}$ phase yields values markedly below those in the $\mathrm{N}$ phase. If referred to the same $T$ these relaxation rates correspond to correlation times that are notably shorter in the RN compared with the $\mathrm{N}$ phase. However, this general interpretation of NMR data remains speculative as long as translational diffusion has not definitely be identified as the process governing the observed relaxation.

Direct evidence for the relation between translational diffusion and NMR data can be provided by the pulsed field gradient NMR (PFG NMR) technique [54] which records molecular displacements typically over a $\mu \mathrm{m}$ range. For example, PFG NMR has been applied to directly assess the diffusion tensor upon entering the $\mathrm{N}$ phase [55]. In these studies diffusion in the direction of the molecules' long axes was found to increase with increasing nematic order. By the same technique the diffusivity of n-alkanes in nanochannels was found to increase with increasing orientational order [56]. These findings are in line with our data where enhanced molecular ordering is accompanied by increasing diffusivities in the direction of $\widehat{\boldsymbol{n}}$. The powerful combination of PFG NMR with magic angle spinning has recently enabled a notable increase in both observation times and gradient pulse intensities [57]. As a consequence PFG NMR diffusion measurements became possible beyond the limits of measurability existing so far. This concerns in particular the first diffusion measurements with liquid crystals confined to nanopores [58]. To stimulate a direct experimental verification of our present predictions using these novel techniques is the primary purpose of this study.

\section{Acknowledgments}

We thank Professor J. Kärger (Universität Leipzig) for his hospitality and fruitful discussions. We also grateful to Lorenzo Guiducci (Max-Planck-Institut für Kolloidund Grenzflächenforschung Potsdam-Golm) for bringing Ref. 27 to our attention and thank the International Graduate Research Training Group 1524 "Self-assembled soft-matter nanostructures at interfaces" for financial support.

\section{Appendix A: Theoretical background}

From a statistical physical perspective the partition function $\mathcal{Q}$ of the canonical ensemble is a key quantity as far as equilibrium systems are concerned. More specifically, it can be demonstrated that with a proper choice of phase space variables, $\mathcal{Q}$ for linear molecules can be written as 31]

$$
\mathcal{Q}=\frac{1}{\Lambda_{\mathrm{th}}^{5 N}}\left(\frac{\mathcal{I}}{m}\right)^{N} \mathcal{Z}
$$

where $\Lambda_{\mathrm{th}} \equiv h / \sqrt{2 \pi m k_{\mathrm{B}} T}$ is the thermal de Broglie wave length,

$$
\mathcal{Z} \equiv \frac{1}{2^{N} N !} \iint \exp [-\beta U(\boldsymbol{R}, \widehat{\boldsymbol{U}})] \mathrm{d} \boldsymbol{R} \mathrm{d} \widehat{\boldsymbol{U}}
$$

is the configuration integral of a system of $N$ indistinguishable molecules, and $\beta \equiv 1 / k_{\mathrm{B}} T$ ( $k_{\mathrm{B}}$ Boltzmann's constant, $T$ temperature). The factor $1 / 2^{N}$ in Eq. A2 corrects for double counting equivalent configurations. These arise because of the head-tail symmetry of the molecules, that is the equivalence of orientations specified by $\widehat{\boldsymbol{u}}_{i}$ and $-\widehat{\boldsymbol{u}}_{i}$. For the isothermal-isobaric ensemble employed in the MC simulations of this work

$$
\Delta \equiv \sum_{\left(s_{\mathrm{x}}, s_{\mathrm{y}}\right)} \exp \left(-\beta P_{\|} A s_{\mathrm{z} 0}\right) \mathcal{Q}
$$

is the relevant partition function [44]. It is then straightforward to show that the Gibbsian potential [4]

$$
\mathcal{G}=-k_{\mathrm{B}} T \ln \Delta
$$

is the relevant thermodynamic potential such that thermodynamic equilibrium is attained if $\mathcal{G}$ is minimum at any fixed values of $N, P_{\|}, T$, and $s_{z 0}$. However, it should be noted that as far as static properties are concerned the specific choice of $m$ or $\mathcal{I}$ does not matter [44]

However, as far as the temporal evolution of the system is concerned the specific choice of both $m$ and $\mathcal{I}$ does matter. To see this let us derive the equations of motion that govern the temporal evolution in a system of linear molecules. Starting from Hamilton's equation of 
motion the translation of the molecular centers of mass is described by

$$
\begin{aligned}
\dot{\boldsymbol{r}}_{i} & =\boldsymbol{v}_{i} \\
\dot{\boldsymbol{v}}_{i} & =\frac{\boldsymbol{f}_{i}}{m}, \quad i=1, \ldots, N
\end{aligned}
$$

where $\boldsymbol{v}_{i}$ and $\boldsymbol{f}_{i}=-\nabla_{\boldsymbol{r}_{i}} U$ are velocity and total force acting on the center of mass of molecule $i$. Similarly, one may derive equations of motion for the rotation of a molecule starting again from Hamilton's equations but this time taking angular velocity $\boldsymbol{\omega}_{i}$ and $\widehat{\boldsymbol{u}}_{i}$ as the conjugate canonical variables rather than linear momentum and center-of-mass position as in the derivation of Eqs. (A5). The resulting expressions are given by

$$
\begin{aligned}
\dot{\widehat{\boldsymbol{u}}}_{i} & =\boldsymbol{\omega} \times \widehat{\boldsymbol{u}} \\
\dot{\boldsymbol{\omega}} & =\frac{\boldsymbol{\tau}_{i}}{\mathcal{I}}, \quad i=1, \ldots, N
\end{aligned}
$$

where $\mathcal{L}=\mathcal{I} \boldsymbol{\omega}[$ see Eq. (1)]

$$
\boldsymbol{\tau}_{i} \equiv \widehat{\boldsymbol{u}}_{i} \times \boldsymbol{g}_{i}=\widehat{\boldsymbol{u}} \times \boldsymbol{g}_{i}^{\perp}
$$

is the torque acting on each molecule, and, following Fincham [59], $\boldsymbol{g}_{i} \equiv-\nabla_{\widehat{\boldsymbol{u}}_{i}} U$ is the so-called "gorque". The gorque may be thought of as a turning "force" that effects changes in molecular orientation. For linear molecules obviously only the gorque $\boldsymbol{g}_{i}^{\perp}$ perpendicular to the molecular axis matters. It is obtained through the projection of $\boldsymbol{g}_{i}$ onto $\widehat{\boldsymbol{u}}_{i}$ via

$$
\boldsymbol{g}_{i}^{\perp}=\boldsymbol{g}_{i}-\left(\boldsymbol{g}_{i} \cdot \widehat{\boldsymbol{u}}_{i}\right) \widehat{\boldsymbol{u}}_{i}
$$

Moreover, in the case of linear molecules the angular velocity must always be perpendicular to the molecular axis, that is $\boldsymbol{\omega}_{i} \cdot \widehat{\boldsymbol{u}}_{i}=0$. This makes it convenient to replace the angular by the orientational velocity $\boldsymbol{w}_{i}$ such that Eqs. A6 can be replaced by the equivalent but, from a numerical perspective, much more convenient equations

$$
\begin{aligned}
\dot{\widehat{\boldsymbol{u}}}_{i} & =\boldsymbol{w}_{i} \\
\dot{\boldsymbol{w}}_{i} & =\frac{\boldsymbol{g}_{i}^{\perp}}{\mathcal{I}}-w^{2} \widehat{\boldsymbol{u}}_{i}, \quad i=1, \ldots, N
\end{aligned}
$$

The derivation of Eqs. A9 requires a little bit of extra but straightforward vector algebra [59]. Finally, Eqs. (A5) and (A9) show that $m$ and $\mathcal{I}$ determine the timescale of translational and rotational motion, respectively.
[1] H. Kleinert, S. Schmidt, and A. Pelster, Phys. Rev. Lett. 93, 160402 (2004).

[2] C. Bechinger, Q.-H. Wei, and P. Leiderer, J. Phys.: Condens. Matter 12, A425 (2000).

[3] M. B. Pinto, R. O. Ramos, and J. E. Parreira, Phys. Rev. D 71, 123519 (2005).

[4] N. Osaka, S. Miyazaki, S. Okabe, H. Endo, A. Sasai, K.I. Seno, S. Aoshima, and M. Shibayama, J. Chem. Phys. 127, 094905 (2007).

[5] J. Dudowicz, J. F. Douglas, and K. F. Freed, J. Chem. Phys. 130, 164905 (2009).

[6] S. M. M. Ramos, A. Benyagoub, B. Canut, and C. Jamois, Langmuir 26, 5141 (2010).

[7] J. Szydlowska, A. Krówczyński, R. Bilewicz, D. Pociecha, and E. Głaz, J. Mater. Chem 18, 1108 (2008).

[8] P. E. Cladis, Phys. Rev. Lett. 35, 48 (1975).

[9] W. K. Lee, B. A. Wintner, E. Fontes, P. A. Heiney, M. Ohba, J. N. Haseltine, and A. B. Smith, Liq. Cryst. 4, 87 (1989).

[10] D. Pociecha, E. Gorecka, and M. Čepič, Phys. Rev. Lett. 86, 3048 (2001).

[11] G. Heppke, H.-S. Kitzerow, D. Lötzsch, and C. Papenfuß, Liq. Cryst. 8, 407 (1990).

[12] G. Sigaud, N. Huu Tinh, F. Hardouin, and H. Gasparoux, Mol. Cryst. Liq. Cryst. 69, 81 (1981).

[13] P. E. Cladis, R. K. Bogardus, W. B. Daniels, and G. Taylor, Phys. Rev. Lett. 39, 720 (1977).

[14] F. Hardouin, G. Sigaud, M. F. Archard, and H. Gasparoux, Phys. Lett. A 71, 347 (1979).

[15] P. E. Cladis, Mol. Cryst. Liq. Cryst. 165, 85 (1988).

[16] L. Longa and W. H. de Jeu, Phys. Rev. A 26, 1632 (1982).
[17] A. N. Berker and J. S. Walker, Phys. Rev. Lett. 47, 1469 (1981).

[18] R. R. Netz and A. N. Berker, Phys. Rev. Lett. 68, 333 (1992).

[19] A. Ferrarini, G. R. Luckhurst, P. L. Nordio, and E. Spoalore, Mol. Phys. 89, 1087 (1996).

[20] E. de Miguel and E. M. del Río, Phys. Rev. Lett. 95, 217802 (2005).

[21] M. G. Mazza, M. Greschek, R. Valiullin, J. Kärger, and M. Schoen, Phys. Rev. Lett. 105, 227802 (2010).

[22] B. J. Borah, H. Jobic, and S. Yashonath, J. Chem. Phys. 132, 144507 (2010).

[23] R. Y. Dong, J. Chem. Phys. 75, 2621 (1981).

[24] R. Y. Dong, J. Chem. Phys. 76, 5659 (1982).

[25] S. Miyajima, K. Akaba, and T. Chiba, Solid State Commun. 49, 675 (1984).

[26] J. Bharatam and C. R. Bowers, J. Phys. Chem. B 103, 2510 (1999).

[27] F. Vollrath and D. P. Knight, Nature 410, 541 (2001).

[28] B. Martínez-Haya, A.Cuetos, S. Lago, and L. F. Rull, J. Chem. Phys. 122, 024908 (2004).

[29] C. Vega and S. Lago, Comput. Chem. 18, 55 (1994).

[30] J. G. Gay and B. J. Berne, J. Chem. Phys. 74, 3316 (1981).

[31] T. Gruhn and M. Schoen, Phys. Rev. E 55, 2861 (1997).

[32] T. Kihara, Adv. Chem. Phys. 5, 147 (1963).

[33] A. Cuertos, B. Martínez-Haya, S. Lago, and L. F. Rull, Phys. Rev. E 68, 011704 (2003).

[34] L. Verlet, Phys. Rev. 159, 98 (1967).

[35] M. Schoen, Physica A 270, 353 (1999).

[36] J. M. Ilnytskyi and M. R. Wilson, Comput. Phys. Commun. 148, 43 (2002). 
[37] H. Pardowitz and S. Hess, Physica A 100, 540 (1980).

[38] R. Eppenga and D. Frenkel, Mol. Phys. 52, 1303 (1984).

[39] W. Maier and A. Saupe, Z. Naturforsch 14a, 882 (1959).

[40] W. Maier and A. Saupe, Z. Naturforsch. 15a, 287 (1960).

[41] A. Richter and T. Gruhn, J. Chem. Phys. 125, 064908 (2006).

[42] M. Schoen and S. H. L. Klapp, Nanoconfined Fluids. Soft Matter Between Two and Three Dimensions (WileyVCH, 2007).

[43] S. H. L. Klapp, Y. Zeng, D. Qu, and R. v. Klitzing, Phys. Rev. Lett. 100, 118303 (2008).

[44] M. Greschek, M. Melle, and M. Schoen, Soft Matter 6, 1898 (2010).

[45] G. W. Robinson, C. H. Cho, and J. Urquidi, J. Chem. Phys. 111, 698 (1999).

[46] J. P. Hansen and I. R. McDonald, Theory of Simple Liquids (Academic Press, 2006).

[47] H. Löwen, Phys. Rev. E 59, 1989 (1999).

[48] M. Karplus and J. N. Kushick, Macromolecules 14, 325 (1981).

[49] L. D. Landau and E. M. Lifshitz, Statistical Physics (Pergamon Press, London, 1980).
[50] Y.-C. Liu, J. D. Moore, Q. Chen, T. J. Roussel, Q. Wang, and K. E. Gubbins, "Diffusion fundamentals, vol. 3," (Leipziger Universitätsverlag, Leipzig, 2009) p. 164.

[51] V. Kukla, J. Kornatowski, D. Demuth, I. Girnus, H. Pfeifer, L. V. C. Rees, S. Schunk, K. K. Unger, and J. Kärger, Science 272, 702 (1996).

[52] T. Kaneko, Y. F. Li, S. Nishigaki, and R. Hatakeyama, J. Am. Chem. Soc. 130, 2714 (2008).

[53] C. Lutz, M. Kollman, and C. Bechinger, Phys. Rev. Lett. 93, 026001 (2004).

[54] R. Valiullin, J. Kärger, and R. Glaser, Phys. Chem. Chem. Phys. 11, 2833 (2009).

[55] S. V. Dvinskikh and I. Furo, J. Chem. Phys. 115, 1946 (2001).

[56] R. Valiullin and A. Khokhlov, Phys. Rev. E 73, 051605 (2006)

[57] W. E. Maas, F. H. Laukien, and D. G. Cory, J. Am. Chem. Soc. 118, 13085 (1996).

[58] E. E. Romanova, F. Grinberg, A. Pampel, J. Kärger, and D. Freude, J. Magn. Res. 196, 110 (2009).

[59] D. Fincham, Mol. Simul. 11, 79 (1993). 